\title{
Ambiguïté de la violence politique : la persécution religieuse durant la guerre civile espagnole (1936-1939)
}

\section{Gabriele Ranzato}

\section{(2) OpenEdition \\ Journals}

\section{Édition électronique}

URL : http://journals.openedition.org/conflits/411

DOI : $10.4000 /$ conflits.411

ISSN : $1777-5345$

Éditeur :

CCLS - Centre d'études sur les conflits lilberté et sécurité, L'Harmattan

Édition imprimée

Date de publication : 15 mai 1993

ISSN : 1157-996X

\section{Référence électronique}

Gabriele Ranzato, «Ambiguïté de la violence politique : la persécution religieuse durant la guerre civile espagnole (1936-1939) », Cultures \& Conflits [En ligne], 09-10 I printemps-été 1993, mis en ligne le 27 janvier 2003, consulté le 30 mars 2021. URL : http://journals.openedition.org/conflits/411 ; DOI :

https://doi.org/10.4000/conflits.411

Ce document a été généré automatiquement le 30 mars 2021.

Creative Commons License 


\title{
Ambiguïé de la violence politique : la persécution religieuse durant la guerre civile espagnole (1936-1939)
}

\author{
Gabriele Ranzato
}

1 Ce qui apparaît particulièrement intéressant dans le phénomène que je m'apprête à illustrer est sa capacité à mettre en évidence l'ambiguïté de la violence politique) ${ }^{1}$. Une ambiguïté qui dérive du fait que cette violence, tout en véhiculant des motivations - et en quelque sorte sa légitimation - à partir de la sphère du politique donc publique, a, originellement, des mobiles d'une autre nature qui, dans une certaine mesure, peuvent se ramener à la sphère de l'affectivité et du privé. Si ceci est vrai en général, la particularité du phénomène consiste dans la qualité spécifique de la violence qui se conjugue dans ce cas avec la violence politique, et que nous pouvons définir dans un premier temps, quoiqu'il s'agisse d'une entité ultérieurement décomposable, comme une violence religieuse.

2 Le phénomène sur lequel on voudrait attirer l'attention est, dans ses grandes lignes, assez connu. Il s'agit de la violence dont furent l'objet les membres de l'Eglise catholique durant la guerre civile espagnole de 1936-39, en particulier dans les premiers mois du conflit. Dans un premier temps, il est bon de l'évaluer pour avoir une mesure de son importance. Le calcul le plus digne de foi, réalisé par Monseigneur Montero Moreno dans un travail publié en 1961 et qui énumère une par une les victimes de cette violence, en spécifiant l'identité, la condition religieuse, la date, le lieu et, souvent, les circonstances de la mort, fournit les chiffres suivants : clergé séculier 4184 victimes, religieux 2365 , religieuses 283 , soit un total de 6832 victimes $^{2}$. Il s'agit d'un bilan nettement inférieur aux chiffres diffusés pendant la guerre; les 16000 victimes, par exemple, dont parle Paul Claudel dans sa fameuse poésie Aux martyrs espagnols dont on peut supposer (même si, sur cet aspect, des évaluations partielles plus récentes en confirment la validité substantielle) qu'ils inclurent des victimes de la guerre et non pas strictement de la persécution ${ }^{3}$. Néanmoins, ces chiffres indiquent un sacrifice d'individus dont la dimension peut apparaître faible au regard du grand 
nombre de victimes de la guerre civile mais qui peut, au contraire, être perçu comme important si l'on considère le montant des pertes par rapport au groupe humain auquel ces victimes appartiennent, c'est-à-dire le pourcentage des pertes par rapport au nombre global des effectifs du clergé.

3 A ce sujet, si l'on parcourt les chiffres relatifs aux différents diocèses, la première chose qui saute aux yeux est que, pour le clergé séculier (le seul pour lequel le calcul en pourcentage fut possible), les assassinats atteignirent dans certains cas la dimension d'une véritable extermination. Dans celui de Barbastro, par exemple, $88 \%$ du clergé fut éliminé, $66 \%$ dans celui de Lérida, $62 \%$ dans celui de Tortosa. Dans d'autres, c'est environ la moitié : $48 \%$ à Malaga, $49 \%$ à Minorca, 55\% à Segorbe, $48 \%$ à Toledo. Mêmes les grands diocèses urbains apparaissent durement touchés, surtout si l'on considère qu'à des pourcentages inférieurs correspondent des chiffres beaucoup plus importants en valeur absolue. A Madrid, 334 prêtres furent tués (30\%), 279 à Barcelone (22\%), 327 à Valence (27\%).

4 Mais l'importance du poids total de la violence exercée sur les membres de l'Eglise au cours de la guerre civile apparait très grande si l'on considère qu'il n'est pas correct d'établir une comparaison entre les victimes de cette violence et les victimes globales de la guerre. La comparaison doit être faite entre eux et ceux qui furent victimes de répression, d'exécution, bref d'une justice - même sommaire - ou de ce que chaque partie considérait comme telle. Sous ce rapport, le bilan des victimes ecclésiastiques apparaît moins faible et, en tout cas, certainement pas sans importance.

5 Ce qui est le plus intéressant à noter est que l'homogénéité présupposée des données confrontées met en lumière leur ambiguïté. Parce que le seul point de vue à partir duquel les données relatives aux victimes ecclésiastiques et aux victimes de la répression politique peuvent être considérées comme homogènes est celui suivant lequel même les premières furent objet d'une violence inspirée par une motivation politique. Or celle-ci est chargée d'ambiguïté, comme on cherchera à le montrer.

6 L'homologation de la violence qui vise les membres de l'Eglise sous la rubrique violence politique fut proposée par certaines des plus hautes autorités de la République, immédiatement après l'explosion de fureur anticléricale. Par exemple, en septembre 1936, une figure éminente du groupe dirigeant républicain, l'ex-ministre de l'agriculture Marcelino Domingo, artisan de la réforme agraire, faisait la déclaration suivante au journal français L'Oeuvre : "Il est vrai que certaines églises ont été brûlées, beaucoup moins que ce qui a été dit. Il est exact qu'en Espagne certains prêtres ont été persécutés, dans une proportion moindre que celle qui s'est dite. ${ }^{4}$ quasiment toutes les églises ont été converties en forteresses, que quasiment toutes les sacristies sont devenues des dépôts de munitions, et la majorité des évêques, prêtres et séminaristes sont devenus des francs-tireurs de la rébellion (...) Si l'Eglise, concluait-il, ne s'était pas alliée à l'armée rebelle, elle aurait pu faire entendre du haut de ses clochers les invocations chrétiennes de paix, au lieu de décharges de guerre, et il n'y aurait pas eu de persécution religieuse" 5 . Ce type de justification fut amplement propagé à travers la presse. "Le 19 juillet, écrivait par exemple le journal barcelonais L'Esquella de la Torratxa, "les militaires traîtres se lancent dans les rues pour instaurer la dictature fasciste. Le peuple, en exposant sa vie dans une tragique explosion de courage viril, écrase la rébellion. Il constate que dans toutes les paroisses il y a des mitraillettes avec lesquelles le peuple est agressé ; que certains couvents se convertissent en petits forts qu'il faut prendre à coups de canons; qu'au milieu des insurgés les prêtres ne 
manquent pas (...) Et le peuple, agité par une légitime indignation, détruit aveuglément ceux qui se sont présentés comme les foyers de la réaction agressive (...) Et maintenant nous voyons ce qui arrive avec la guerre. Arme au bras, prêtres, frères, jésuites et séminaristes combattent le peuple. Les évêques et même le pape de Rome bénissent les rebelles. Le Vatican leur ouvre ses coffres. Les jésuites leur versent l'argent dont ils ont besoin. Le cri "Vive le Christ Roi !" résonne dans les rangs des traîtres !" 6.

7 Même si les prises de position de l'Eglise en faveur des militaires rebelles semblaient être parfaitement cohérentes avec ces comportements de belligérance qui lui sont attribués, les accusations de participation proprement "militaire" étaient en réalité infondées, se limitant à quelques rares épisodes à la dynamique incertaine ${ }^{7}$. Mais audelà des circonstances de fait, il restait comme explication, et justification, de cette violence exercée contre le clergé une motivation politique : l'Eglise est ennemie de la République, l'Eglise s'est rangée du côté des militaires rebelles contre le peuple qui s'opposait au coup d'Etat, et donc contre la République; le peuple, en son nom, frappe ses ennemis et parmi eux, justement, aussi les membres de l'Eglise.

8 Certes il est bien clair que cette explication a quelque fondement parce qu'au-delà des prétendues justifications relatives à l'intervention armée du clergé, il est indubitable que la responsabilité de l'Eglise espagnole, dans le passé proche et plus lointain, son indifférence fondamentale aux conditions des classes les plus pauvres et, au contraire, sa solidarité avec les classes dominantes, son soutien aux formules politiques les plus antidémocratiques, sa belligérance non armée contre la République ont toujours davantage exaspéré la haine anticléricale, qui aurait ensuite explosé quand les forces réactionnaires, alliées traditionnelles de l'Eglise, allaient tenter d'étouffer dans le sang la victoire du Front populaire. Comme le problème qui se pose est celui de la nature de cette violence, il importe peu d'objecter qu'au fond l'Eglise espagnole était réactionnaire de façon moins homogène qu'on ne le présente et que la viser globalement fut une erreur politique, à l'exclusion du cas basque.

Seulement cette motivation politique est moins claire qu'il apparaît de prime abord. L'indice d'une plus grande complexité de cette motivation réside dans l'expression "persécution religieuse" utilisée par Marcelino Domingo et qui, par la suite, sera largement employée pour désigner le phénomène. Déjà, le terme même de "persécution" renvoie à une explication politique de la violence plutôt discutable. Cependant il s'agit d'un terme tout à fait approprié pour désigner le phénomène. Si, en effet, l'on doit entendre par persécution une violence systématique exercée sur les membres d'un groupe humain, plus ou moins motivée, mais qui, pour être exercée, a comme condition suffisante la seule appartenance au groupe, indépendamment de toute responsabilité personnelle, il est indubitable que, dans l'Espagne de l'époque, les membres de l'Eglise furent l'objet de persécution.

Parmi les nombreux témoignages allant en ce sens, celui de Hilari Raguer, homme d'Eglise résolument antifranquiste, est particulièrement significatif. Il a écrit dans son livre La espada y la cruz : "Bien que le nombre et les circonstances ${ }^{8}$ aient été exagérés, bien que ce qui s'est publié sur cette question soit très abondant et ait bénéficié de circonstances politiques qui lui ont donné une très grande divulgation (...), il faut pourtant admettre cette terrible réalité historique: pendant de nombreux mois, il suffisait que quelqu'un soit identifié comme prêtre, religieux ou simplement membre d'une congrégation ou d'un mouvement apostolique, pour qu'il soit exécuté sans procès"s. 
11 Le caractère indiscriminé de la violence qui s'abat sur le clergé espagnol est rendu particulièrement évident dans le fait que même ces minorités ecclésiastiques qui s'étaient distinguées par une plus grande compréhension des revendications populaires et une pleine adhésion à la République en furent l'objet. Par exemple cette violence "touche particulièrement ces ecclésiastiques qui avaient le plus travaillé en faveur d'une Eglise catalane plus ouverte et plus progressiste (...) Des milliers de catalans démocrates persécutés pour leur catholicisme ont dû chercher refuge dans l'Espagne franquiste" ${ }^{110}$.

12 S'il s'est agi de persécution, elle peut certainement être considérée comme une violence politique, et elle l'est d'autant plus dans un cas comme celui-ci où la persécution poursuit un objectif d'extermination, puisque les persécuteurs se proposent de libérer la collectivité d'un groupe considéré comme néfaste pour elle. Mais nous savons que ceci est généralement l'enveloppe d'un besoin psychologique collectif de projeter en-dehors de soi le mal total et de recouvrer une sécurité dans l'effort de purifier, de rendre limpide et immédiatement reconnaissable sa propre identité. Un effort d'autant plus exaspéré dans une guerre civile où l'anxiété qui dérive de l'impossibilité de reconnaître l'ennemi, ou encore de la menace d'une "cinquième colonne" constamment aux aguets, peut trouver une libération dans la persécution de l'unique "ennemi" qui ne se rencontre pas sur le champ de bataille, qui ne revêt pas un uniforme mais qui est clairement individualisable par des signes extérieurs assez évidents, comme le sont ceux des membres de l'Eglise.

Ce n'est pourtant pas l'aspect de la persécution qui met le plus en évidence l'ambiguïté de la violence qui s'est abattue sur le clergé au cours de la guerre civile espagnole mais plutôt l'adjectif qui l'accompagne, c'est-à-dire le caractère "religieux" de cette persécution, en tant qu'elle présente des caractères qui, de toute évidence, n'appartiennent pas au champ du politique. A ce propos, on ne peut pas ne pas relever que chaque réflexion sur cet aspect doit tenir compte du fait que les sources parmi lesquelles la plus importante, la Causa General, c'est-à-dire l'instruction générale sur ce que l'on appelait les "crimes rouges" réalisée dans l'après guerre par le régime franquiste ${ }^{11}$ sont inévitablement très partiales. Car les témoignages et la documentation sur les persécutions sont, en grande partie, le fait des persécutés tandis que les persécuteurs préfèrent nier ou se taire.

Ici, les intentions de propagande du régime franquiste, l'auto-valorisation des ordres religieux ou des sièges épiscopaux, non exemptes de finalité de canonisation concernant certaines victimes de la persécution, facilitent fréquemment une version hagiographique du phénomène où sont évidentes une forte volonté d'exaltation des victimes de la persécution et, en même temps, une forte volonté de dénigrement des persécuteurs. Cependant même dans ces limites, les sources dont nous disposons mettent en évidence certains caractères indiscutables qui intéressent notre propos.

Il y a en premier lieu, comme caractère fort et diffus du phénomène, l'aspect iconoclaste. La destruction des églises, édifices religieux et images sacrées acquiert immédiatement, dès les premiers jours de la guerre civile, une dimension si fortement symbolique pour marquer le choix du camp qu'il n'y eut quasiment pas de centre habité resté sous le contrôle de la République où ne se soit pas réalisée la destruction de la grande partie, sinon de la totalité des lieux et des objets de culte. Quelques chiffres à titre d'exemple: dans le diocèse de Valence, 800 églises sont indiquées comme totalement rasées, 354 dans celui d'Oviedo. Dans de nombreux autres diocèses, il n'y a 
pas d'édifices religieux qui n'aient été partiellement détruits, profanés ou saccagés ; ainsi à Almeria, Barbastro, Ciudad Real, Segorbe et Tortosa. Dans d'autres, comme Barcelone, Cartagêne, Madrid, Orihuela, Santander et Tolède, les églises restées indemnes sont en nombre très limité ${ }^{12}$.

Les sources de ces données sont en grande partie constituées par des témoignages recueillis par la Causa General et des rapports de diocèses de l'après-guerre. Elles inspirent pourtant une certaine méfiance. Mais des études plus récentes, d'origine non cléricale, confirment également l'importance du phénomène. Selon l'une d'entre elles par exemple, seules deux modestes chapelles de la ville de Gerona furent totalement épargnées de toute espèce d'assaut et de spoliation. Toutes les autres furent intégralement ou partiellement dévastées ou saccagées et, pour une grande part, elles furent destinées à d'autres fonctions : magasins, garages, écoles, prisons, musées - on a réussi sous cette forme à préserver la cathédrale de la destruction -, hospices, cantines, casernes, etc. Deux d'entre elles furent incendiées et plus tard totalement démolies; l'église paroissiale de Domeny, située en ville, explosa à la dynamite ${ }^{13}$.

17 A la différence de ce qu'il est plus facile d'imaginer, la destruction des églises ne fut pas souvent l'effet d'une irrésistible furie populaire mais une opération systématique, délibérée, des autorités républicaines locales comme s'il s'agissait d'un simple acte administratif. A ce propos, il est intéressant de lire par exemple le procès-verbal d'une réunion du Conseil Communal de Castellon, chef-lieu de province de la région du Levante, consacrée à l'opportunité de la démolition de la Iglesia Mayor démolition qui fut effectivement réalisée par la suite où, dans le cadre d'un accord général sur la nécessité d'une telle opération, se joignent aux motivations d'ordre urbain les motivations présentées par certains conseillers qui soutiennent textuellement que "les raisons morales de la démolition de l'église sont plus importantes que celles d'ordre matériel ${ }^{14}$ cette baraque représente quelque chose de tellement infâme qu'il est absolument urgent de procéder à sa démolition"15.

La signification attribuée aux destructions des lieux de cultes et des images sacrées était tellement forte que, d'après les témoignages, on incendiait l'église paroissiale dans certains endroits davantage pour éviter d'être suspectés par les comités révolutionnaires d'autres localités, d'une adhésion trop timide à la cause républicaine que sous une impulsion forte et authentique à accomplir cette action. C'est le cas, par exemple, à Urràcal (Almeria) où, selon le curé, les membres du Comité révolutionnaire local dont le "but principal était de démontrer aux éléments étrangers qui pouvaient venir au pays qu'ils avaient réquisitionné la maison paroissiale", échangèrent la clé de cette dernière contre la promesse de prévenir le prêtre à temps au cas où les "éléments étrangers" seraient venus le chercher pour le mettre dehors ${ }^{16}$. Des épisodes analogues se sont produits, dans la même province, à Castro de Filabres et à Vélez Rubio où, selon le curé, "la destruction des images fut due aux pressions des éléments étrangers et à la faiblesse des gens du pays qui craignaient de rencontrer des problèmes plus graves s'ils ne consentaient pas à leur prétention" ${ }^{17}$.

19 Même les assassinats des prêtres, par leur fréquence, peuvent avoir revêtu la signification symbolique d'un brevet révolutionnaire et, également, la fonction d'un don sacrificiel de la communauté, qui la mettait à l'abri de "problèmes plus graves". Une telle hypothèse semblerait validée par les nombreux cas de villages où l'unique victime de la révolution fut justement le curé. Cette thèse pourrait par exemple être étayée par un témoignage annexe à la Causa General de la province de Huesca dont 
l'auteur se félicite du fait que, dans son pays, "bien qu'il fût pendant vingt mois sous la domination rouge, aucun assassinat de personnes du lieu n'avait été commis, et même la vie du curé avait été respectée" ${ }^{18}$.

Tant les destructions d'églises - comme exécution d'une décision administrative - que les assassinats de prêtres - surtout ceux, dont abondent les témoignages de la Causa general, commis contre l'habit et non contre la personne - pourraient être considérés comme des violences purement politiques, au moins dans la mesure où l'anticléricalisme peut être considéré comme une option proprement politique. Mais la frontière entre ce qui est anticlérical et ce qui est antireligieux, et ce qui appartient en tant que tel à la sphère du religieux et non du politique, est très ténue. Dans les causes de béatification des religieux tués durant la guerre civile espagnole, conservées dans les Archives Secrètes du Vatican - cent dix huit ont été instruites - les témoins insistent beaucoup sur les déclarations des meurtriers selon lesquelles l'acte qu'ils commettaient était motivé essentiellement par la haine de la religion ${ }^{19}$. Mais il est légitime de se méfier de tels témoignages car la motivation antireligieuse est une condition sine qua non du procès de béatification pour cause de martyre, comme le sont tous ceux relatifs à la guerre d'Espagne.

21 Nous avons pourtant de nombreux autres indices du fait que ces actes de violence ne s'épuisent pas dans le mobile politique. Arrêtons-nous, par exemple, sur le phénomène de destruction des images. Même dans ce cas nous pouvons y voir un geste politique de destruction symbolique. La destruction d'un Christ ou d'une Vierge peut, d'une certaine manière, être considérée comme celle d'une croix gammée ou d'une faucille et d'un marteau. Mais si nous approfondissons et nous arrêtons sur les modalités de la destruction, le sens politique s'estompe pour laisser place à d'autres significations.

La destruction est en effet souvent précédée de mutilations ou d'autres faits profanateurs. Parmi les plus fréquents, nous trouvons par exemple l'ablation (dans le cas de statues) ou le percement (dans le cas de tableaux) des yeux des images . ${ }^{20}$, l'amputation, ou mieux, l'imitation d'une amputation, des organes sexuels masculins ${ }^{21}$, le fait de traîner les statues à travers les rues ${ }^{22}$, comme l'on faisait d'ailleurs parfois avec le cadavre du prêtre ${ }^{23}$, les coups portés aux effigies saintes avec des fouets et des bâtons ${ }^{24}$. Cette personnification des images saintes, qui atteste l'inassouvissement du geste symbolique, rejoint parfois des niveaux extrêmes dans lesquels, sous le comportement moqueur et offensif contre les croyants, transparaît le besoin d'une communication, même sous une forme agressive, avec la divinité. Tel est le cas quand la statue du Christ ou d'un saint est portée dans l'étable ou parmi les porcs, quand elle est conduite au pâturage ou à l'abreuvoir, quand elle est portée à l'auberge pour "boire du vin", ou quand elle est affublée d'habits burlesques, souvent de soldats, ou encore mise en sentinelle, munie d'un balai ou d'un bâton, sur le parvis d'une église ou dans une rue publique ${ }^{25}$.

23 Ces mises en scène grossières sur les images sacrées sont à la frontière de la simple iconoclastie et d'une autre famille de phénomènes assez diffus qui connotent la persécution religieuse, c'est-à-dire les parodies de rites catholiques, faites de processions des images destinées au feu, d'imitations burlesques de la liturgie avec les ornements sacrés, de pantomimes tragiques ou carnavalesques ${ }^{26}$ d'épisodes évangéliques qui, souvent, accompagnent l'action destructrice ${ }^{27}$. Mais plutôt que de moqueries et de dérision qui seraient une fin en soi, ces parodies dans lesquelles on rencontre une prédilection particulière pour les scènes de la Passion, le moment de 
culte le plus choral et envoûtant du catholicisme espagnol, ont le caractère du contrerite. Elles s'expriment ainsi dans un langage symétrique à celui du rituel catholique ; un langage stratifié dans la culture populaire, appris, directement ou indirectement, àtravers des siècles de fêtes, de représentations sacrées, d'autodafés ou, vice-versa, dans les contre-rites déjà englobés dans cette culture religieuse ${ }^{28}$.

Mais peut-être que l'indicateur le plus significatif d'un mobile non politique de l'ensemble de ces actes de violence dirigés contre l'Eglise dans la zone républicaine est la profanation des cimetières catholiques et des tombes conservant les corps des ecclésiastiques. Il s'agit d'un phénomène qui a déjà attiré l'attention du chercheur américain Bruce Lincoln ${ }^{29}$ mais qui, à travers les rapports des diocèses recueillis dans la Causa General, apparaît beaucoup plus répandu que les épisodes limités, documentés photographiquement, qui concernent Barcelone et Madrid ${ }^{30}$. La multiplicité des cas, leur diffusion géographique - des manifestations analogues ont lieu dans la province d'Almeria comme dans celle de Castellon, à Oviedo comme à Guadalajara ${ }^{31}$ - indiquent qu'ils ne doivent pas être considérés comme des aberrations circonscrites mais comme les manifestations de la complexité du phénomène. Et ce d'autant plus que, au-delà de la particularité de chaque épisode, certaines caractéristiques apparaissent communes à tous.

Il y a d'une part la volonté destructrice qui ne se satisfait pas de la fin de la vie de son objet mais veut le mortifier au-delà de la mort. Elle doit s'acharner sur les dépouilles mortelles, les traîner, les décomposer, les désintégrer; mais elle doit détruire également l'image, le corps-symbole, s'en moquer, en montrer la misère. Dans cette perspective, le sort réservé aux cadavres fut quelquefois le démembrement et leur mélange avec d'autres jusqu'à leur faire perdre toute identité résiduelle ${ }^{32}$; ou alors leur exhibition sous une forme moqueuse, dans la plupart des cas en guise d'épouvantails monstrueux $^{33}$. D'autre part, se poursuit la démonstration, concrète et symbolique, de la mortalité, de l'impuissance et de la corruption de l'Eglise. A cette fin les cadavres sont exposés à différents points de la ville et montrés à la foule dans leurs divers états de décomposition ${ }^{34}$. Ils indiquent à tous que ces corps soi-disant saints subissent le sort de tous les autres, qu'aucune prédilection divine particulière n'a pu les soustraire ni à la mort ni à l'horreur qu'ils ont ensuite été contraints de subir. En outre, en se révélant dépourvus d'intégrité, non seulement ils se révèlent dépourvus de sainteté mais surtout ils manifestent que la pourriture à laquelle ils sont réduits est le miroir de leur pourriture morale ${ }^{35}$.

Une violence qui touche les tombes et les cadavres montre, d'un côté, que le procès de civilisation décrit par Elias, notamment son indicateur: le seuil de tolérance au spectacle de la souffrance et du corporel (il n'y a rien de plus terriblement corporel que la décomposition du corps) n'est pas un processus linéaire ni constamment en progrès. D'un autre côté, une violence comme celle qui vient d'être décrite, dont le caractère non politique est évident, jette un éclairage sur les autres violences qui l'ont accompagnée et dont les significations apparaissent alors plus complexes. L'assassinat d'un prêtre, qui peut être un simple geste politique, acquiert ainsi un caractère d'ambiguïté et une signification plus complexe dans le contexte d'ensemble des violences repérées : iconoclastie, contre-rites, profanations de tombes. La recherche d'une définition plus précise de cette signification dépasse les limites du sujet traité ; cependant il est évident qu'une telle réflexion ne peut pas rester circonscrite aux 
raisons non politiques de la violence politique mais doit s'élargir aux raisons non politiques de la politique tout court ${ }^{36}$.

\section{NOTES}

1. Traduit de l'italien par Isabelle Sommier.

2. A. Montero Moreno, Historia de la persecucion religiosa en Espana 1936-1939, Madrid, 1961, p 762.

3. En 1937, Juan Estelrich publiait à Paris un livre intitulé La persécution religieuse en Espagne, dans lequel il parlait de 16.750 prêtres assassinés ( $p 76$ ). Ce chiffre, repris également par Paul Claudel dans un vers fameux - "16.000 prêtres et pas même une apostasie !" - de sa poésie Aux martyrs espagnols, constitue la base, plus ou moins amplifiée, de nombreuses oeuvres de propagande contemporaines et postérieures. Le fonds de l'ambassadeur de Franco auprès du Saint Siège, Yanguas Messia, et de son ministre des affaires extérieures, le Comte de Jordana indique le même chiffre. Cf. A. Marquina Barrio La diplomacia vaticana y la Espana de Franco (1936-1945), Madrid, 1983, p 417 et 429.

4. Mais d'autre part le gouvernement a constaté que

5. L'Oeuvre du 30 septembre 1936.

6. Cité dans J. Massot "Els catolics catalans durant la guerra civil" in Questions de vida cristiana, $\mathrm{n}^{\circ}$ 131-132, 1986, p 61-62.

7. L'unique épisode confirmé d'"église-petit fort" fut, dans la région catalane, celle de l'église des Carmélites de la Diagonal de Barcelone où se barricadait un groupe de militaires insurgés. Sur cette question, voir le livre de A. Manent et J. Raventos, L'església clandestina a Catalunya durant la guerra civil (1936-1939), Montserrat, 1984, p 34-38. La crédibilité des accusations contre l'Eglise se fonde surtout sur la mémoire historique des prêtres et des frères guérilleros de la guerre d'Indépendance et surtout des guerres carlistes, théatre des hauts faits des curas trabucaires (prêtres avec le fusil). A ce propos, voir J. Caro Baroja, Introduccion a una historia contemporànea del anticlericalismo espanol, Madrid, 1980, p 171 et ss.

8. de la persécution religieuse

9. H. Raguer, La espada y la cruz, Barcelona, 1977, p 147.

10. J. Benet "Un trauma per a la Església catalana", in Questions de vida cristriana, $1986, \mathrm{n}^{\circ} 131-132, \mathrm{p}$ 9-10.

11. La Causa General fut promue par le gouvernement franquiste en avril 1940 par un décret qui attribuait au Procureur Général du Tribunal Supremo les pouvoirs pour préparer une instruction d'information sur les délits commis dans la zone rouge. Divisée en onze rubriques, dont une consacrée spécifiquement à la persécution religieuse, la Causa General se conclut sur un réquisitoire à caractère ouvertement propagandiste et d'une valeur modeste par rapport à la volmineuse documentation recueillie. Cette dernière, composée de 950 fascicules, est conservée à l'Archivo Historico Nacional de Madrid.

12. Cf. A. Montero, op. cit., p 629-630. 
13. Cf. J. Busquets "La destruccio d'esgliésies a la ciutat de Girona el 1936 i les seves excepcions", in A.A. et V.V, La guerra civil a les comarques gironines (1936-1939), Girona, 1986, p 189 et ss.

14. puisque

15. Archivo Historico Nacional (AHN), Causa General (CG), Castellon, dossier 1406 (1).

16. AHN, CG, Almeria, f. 1164 (1).

17. ibidem.

18. AHN, CG, Huesca, f. 1415 (1). Souligné par moi.

19. Le nombre des procès engagés a été calculé en consultant la Sacra Congregatio pro causis sanctorum Index ac status causarum, Roma, 1985. Les actes d'instruction et positiones conservés aux Archives Secrètes du Vatican ne peuvent pas être cités.

20. Des exemples de ce type se trouvent au monastère de San Cugat del Vallés (AHN, CG, Barcelone, f. 1674), à Oria et Cuevas de Almanzora (AHN, CG, Almeria, f. 1164 -1-). Pour ce qui concerne la banlieue de Madrid de Carabanchel Bajo, un témoinagne affirme : "La maitresse du pays, accompagnée par les enfants, jette à terre les images qui se trouvaient dans l'église et en grève les yeux avec les clous" (AHN, CG, Madrid, f. $1557-2-)$.

21. Des mutilations de ce type sont signalées, par exemple, à Brihuega (AHN, CG, Guadalajara, f. 1243) et San Cugat (AHN, CG, Barcelona, f. 1674).

22. Des épisodes de ce genre, qui en général précèdent l'immolation des statues par le feu, sont signalés par exemple à Almeria (AHN, CG, Almeria, f. 1164 -1-), Alcocer (AHN, CG, Guadalajara, f. 1070 -1-), Avilés et Candàs (AHN, CG, Oviedo, f. 1343 -1-), Argecilla et Trillo (AHN, CG, Guadalajara, f. 1243).

23. Dans un témoignage sur l'assassinat du prêtre de Vilanova de la Roca (barcelone), on dit que ses assassins le trainèrent par les rues en offrant aux passants de la viande de porc (AHN, CG, Barcelona, f. 1677). Un témoignage analogue est rapporté par Fogàs de tordera (AHN, CG, Barcelone, f. 1674).

24. Des cas de fustigation publique de statues sacrées sont mentionnés par Cuevas de Almanzora, San Sebastian, Arboleas (AHN, CG, Almeria, f. 1164 -1-) et Udrion de Trubia (AHN, CG, Oviedo, f. 1343 -1-)

25. Des exemples de déguisements ont été enregistrés, entre autres, à Seron (AHN, CG, Almeria, f. 1164 -1-) et Alcocer (AHN, CG, Guadalajara, f. 1070 -1-). L'épisode d'un Christ conduit à l'étable et à l'abreuvoir aurait eu lieu à Lucainena de las Torres (AHN, CG, Almeria, f. 1164 -1-) ; l'épisode de la statue de San Roque conduite à la taverne pour boire du vin est rappelé par le prêtre de San Esteban de Litera (AHN, CG, Huesca, f. 1415 -2-), qui voit un exemple de "châtiment divin" dans le fait que l'auteur de l'acte sacrilège serait mort peu après noyé dans un canal.

26. En ce sens, l'épisode de Morillejo (Guadalajara), relaté par un curé, est particulièrement significatif : "Dans l'église on a palabré de la chaire contre tout ce qui est divin et humain et après, on a simulé une procession en habillant un âne avec les parements sacrés." (AHN, CG, Guadalajara, f. 1070 -1-).

27. "A Blanca, écrit Montero Moreno, durant la nuit où eurent lieu les incendies, une procession burlesque s'organise dans le pays, avec l'image d'un Christ gisant et le trône du Saint Sépulcre. Couverts de chapes et de chasubles, les miliciens entonnaient des chansons dérisoires autour des images qui furent ensuite jetées dans un feu de joie." (A. Montero Moreno, Historia..., op. cit., p 645). Des épisodes analogues sont très nombreux dans la Causa General. Mentionnons simplement quelques localités où ils se sont vérifiés. Province de Barcelone : Roda de Ter, Voltregà, Palleja, San Quirico de 
Tarrasa, Frontanyà. Province de Castellon : Almazora. Province d'Almeria : Benahadux, Terque, Los Gàzquez, Almeria, Alquién, Los Molinos de Viento, Alcolea. Province d'Ovideo : Lugo de Llanera, Ujo. Province de Guadalajara : Morillejo, Canredondo. Province de Gerona : Palau Sabardera, Espolla.

28. Un exemple intéressant du caractère antirituel de certaines manifestations inscrites dans la tradition religieuse du pays peut s'observer dans la fête du jeudi saint de Leon, où, sous le nom de El entierro de Genarin, se déroule une véritable parodie de la Passion du Christ (Cf. M. Tate "Tradicion y humorismo en la Semana Santa de la Ciudad de Leon", in L. Diaz, Etnologia y folklore en Castilla y Leon, Salamanca, 1986, p 155-166. 29. "Revolutionary exhumations in Spain, July 1936", in Comparatives Studies in Society and History, $\mathrm{n}^{\circ}$ 2, 1985.

30. Outre l'article de Lincoln, une documentation photographique intéressante sur la profanation des tombes est contenue dans J. Estelrich La persecution..., op. cit. ; A. De Castro Albarràn La gran victima (la iglesia espanola victima de la revolucion roja), Salamanca, 1940 ; J. Perez de Urbel Los màrtires de la Iglesia (testigos de su fe), Barcelone, 1956.

31. D'après un premier examen incomplet de la documentation relative à la persécution religieuse recueillie pour la Causa General, il résulte que, outre Madrid et Barcelone, des épisodes de profanation de tombes se vérifient à Solsona, Rupit, Canovellas, Manresa, Serrateix (Barcelone) ; Benicarlo (Castellon) ; Urgel et Bellcaire de Urgel (Lérida) ; Almeria et Albanchez (Alméria) ; Cenero, Avilès, Las Caldas, San Felix de Valdesoto, Argüelles de Siero (Oviedo) ; San Sebastian (Guipùzcoa) ; Alcocer, Fuensavinan et Valdeanchita (Guadalajara)

32. "Dans l'église des pères franciscains (d'Avilès), toutes les sépultures cassées et les restes (des cadavres) furent répandus à terre" (AHN, CG, Oviedo, 1343-1-). "Tombes et sarcophages furent profanées et le dallage de l'église (paroisse de Serrateix) et du cloître fut converti dans un cadre macabre de crânes et d'os" (AHN, CG, Barcelone, 1647-2-). "Ils profanèrent les tombes en semant les restes sur tout le dallage" (AHN, CG, Lérida, 1471 -1-).

33. "Ils soulevèrent le magnifique carrelage de marbre de l'église (de san Pietro Apostolo d'Almeria) et les cadavres des religieux san franciscains et des personnes appartenant aux familles qui avaient droit à la sépulture dans l'église, et les cadavres momifiés furent exposés à la porte du temple avec des bérets et des allégories marxistes à la risée de la foule" (AHN, CG, Almeria, 1164 -1-). "Dans le couvent des soeurs (d'Alcocer), le corps de la fondatrice fut exhumé et exposé durant plusieurs jours au public, aux risées et aux moqueries du petit peuple barbare" (AHN, CG, Guadalajara, 1070 -1-).

34. La documentation photographique relative à l'exposition des cadavres des religieuses salésiennes de Barcelone, plusieurs fois reproduite, est impressionnante de ce point de vue. Voir la note 26.

35. Parmi les mobiles de profanateurs de tombes, il y a parfois le désir de prouver les crimes, souvent liés à des relations sexuelles illicites, des religieux des deux sexes. A Madrid, en 1936, les photos de deux cadavres de soeurs de l'Eglise des Carmélites, avec dans leur tombe ceux de nouveaux nés, furent publiées, pour démontrer leurs amours illicites, dans le journal $\mathrm{ABC}$, provoquant l'embarras du gouvernement républicain qui les fait immédiatement disparaitre (Reporté dans A. Montero Moreno, op. cit., p 431, note 3). Nous trouvons des indices analogues dans la Causa General : "Les tombes de l'église (de Rupit) furent ouvertes et les cadavres extraits, quelqu'un soutenait que les 
enfants des ecclésiastiques y étaient enterrés" (AHN, CG, Barcelone, 1674) ; "Pour provoquer la fureur de la foule, ils propagèrent à travers la ville la nouvelle qu'à côté des cadavres des religieuses carmélites exhumés de la chapelle du Santissimo de l'église paroissiale (de Nuestra Senora del carmen di Manresa), il y avait des cadavres d'enfants et de jeunes filles assassinés par les prêtres" (AHN, CG, Barcelone, 1647-2). Pour les antécédents de cet aspect de l'agressivité anticléricale, comme d'ailleurs pour un examen de la persécution religieuse de 1936-39 dans une perspective de longue durée, nous renvoyons à notre travail "Dies Irae : la persecuzione religiosa nella zona repubblicana durante la guerra civile spagnola (1936-1939)", in Movimento Operaio e Socialista, $\mathrm{n}^{\circ} 2,1988$, p 195-220.

36. En français dans le texte

\section{RÉSUMÉS}

L'exemple des violences dirigées contre les ecclésiastiques pendant la guerre d'Espagne (1936-1939) souligne la difficulté de cerner précisément les frontières d'une violence politique par opposition à d'autres catégories d'analyse : violences religieuses, violences iconoclastes...Les systèmes de " légitimation " allégués, la nature et la distribution des actes de violences opérés permettent d'entrevoir la multidimensionnalité de ce qui se joue dans ces situations extrêmes : argument de nécessité, dépréciation des victimes dans le camp républicain à quoi s'oppose du point de vue de l'Eglise catholique une stratégie d'éclairage purement religieux.

The example of violence against members of the church during the Spanish Civil War (1936-1939) shows the difficulty of defining different categories of political violence : religious violence, dissident violence, etc. The systems of " justification ", the distribution and nature of the acts of violence reflect the multidimensional character of such extreme situations : the argument of " need ", the depreciation of the victims by the Republicans confronted by the purely religious approach favoured by the Catholic Church.

\section{INDEX}

Mots-clés : guerre, histoire, religion, répression, violence

Index géographique : Espagne

Index chronologique : 1930 - 1940 